PROCEEDINGS OF THE

AMERICAN MATHEMATICAL SOCIETY

Volume 126, Number 9, September 1998, Pages 2541-2548

S 0002-9939(98)04321-4

\title{
THE MAXIMUM CONDITION ON ANNIHILATORS FOR POLYNOMIAL RINGS
}

\author{
FERRAN CEDÓ AND DOLORS HERBERA \\ (Communicated by Ken Goodearl)
}

\begin{abstract}
For each positive integer $n$, we construct a commutative ring $\mathcal{R}$ such that the polynomial ring $\mathcal{R}\left[x_{1}, \ldots, x_{n}\right]$ satisfies the maximum condition on annihilators and $\mathcal{R}\left[x_{1}, \ldots, x_{n+1}\right]$ does not. In particular, there exists a commutative Kerr ring $\mathcal{R}$ such that $\mathcal{R}[x]$ is not Kerr. This answers in the negative a question of Faith's.
\end{abstract}

\section{INTRODUCTION}

All rings in this paper are commutative. We say that a ring $R$ has ascending chain condition on annihilators (acc $\perp$ ) if it satisfies the maximum condition on annihilators (of subsets of $R$ ). Recall that a ring $R$ is Goldie if it has acc $\perp$ and has no direct sum of infinitely many non-zero ideals. In [3] Kerr constructs a Goldie ring $R$ such that $R[x]$ does not satisfy acc $\perp$. Following [2], we say that a ring $R$ is Kerr if $R[x]$ has acc $\perp$.

In [1], Camillo and Guralnick show that if $R$ is an algebra over an uncountable field $K$ satisfying acc $\perp$ then $R[x]$ satisfies acc $\perp$; thus, if $R$ is a Kerr algebra over an uncountable field $K$ then $R[x]$ is Kerr. On the other hand, in [4] Roitman proves that for any countable field $K$ there exists a $K$-algebra $R$ satisfying acc $\perp$ such that $R[x]$ does not satisfy acc $\perp$. In [2], Faith asks: If $R$ is a Kerr ring, is $R[x]$ ? We use Roitman's techniques [4] to construct for each non-negative integer $n$ and for each countable field $K$ a $K$-algebra $\mathcal{R}$ such that $\mathcal{R}\left[x_{1}, \ldots, x_{n}\right]$ satisfies acc $\perp$ and $\mathcal{R}\left[x_{1}, \ldots, x_{n+1}\right]$ does not. This answers Faith's question in the negative. For $n=0$, $\mathcal{R}$ is the example constructed by Roitman in [4].

Our ring $\mathcal{R}$ is semiprimary, with index of nilpotency 3, and has infinite Goldie dimension. We do not know whether for any countable field $K$, there exists a Goldie $K$-algebra $R$ such that $R[x]$ is not Goldie. We remark that Kerr's example is a $\mathbb{Z} / 2 \mathbb{Z}$-algebra.

\section{Preliminary lemmas}

Let $K$ be a field. Let $u_{\alpha_{1}, \ldots, \alpha_{n+1}}, v_{\beta_{1}, \ldots, \beta_{n+1}}, x_{1}, \ldots, x_{n+1}$ be indeterminates over $K$, where $\alpha_{i}, \beta_{i}, n$ are non-negative integers, $0 \leq \alpha_{1}+\cdots+\alpha_{n+1} \leq m$,

Received by the editors May 10, 1996 and, in revised form, January 30, 1997.

1991 Mathematics Subject Classification. Primary 16P60, 13B25.

Both authors are partially supported by the DGICYT (Spain), through the grant PB95-0626, and by the Comissionat per Universitats i Recerca de la Generalitat de Catalunya.

(C)1998 American Mathematical Society 
$0 \leq \beta_{1}+\cdots+\beta_{n+1} \leq p$ and $m, p \geq 1$. Set

$$
\begin{aligned}
& U=\left\{u_{\alpha_{1}, \ldots, \alpha_{n+1}} \mid 0 \leq \alpha_{1}+\cdots+\alpha_{n+1} \leq m\right\} \\
& V=\left\{v_{\beta_{1}, \ldots, \beta_{n+1}} \mid 0 \leq \beta_{1}+\cdots+\beta_{n+1} \leq p\right\}
\end{aligned}
$$

Let $B=K[U, V]$. Let

$$
\begin{aligned}
& f\left(x_{1}, \ldots, x_{n+1}\right)=\sum_{\alpha_{1}+\cdots+\alpha_{n+1}=0}^{m} u_{\alpha_{1}, \ldots, \alpha_{n+1}} x_{1}^{\alpha_{1}} \cdot \ldots \cdot x_{n+1}^{\alpha_{n+1}}, \\
& g\left(x_{1}, \ldots, x_{n+1}\right)=\sum_{\beta_{1}+\cdots+\beta_{n+1}=0}^{p} v_{\beta_{1}, \ldots, \beta_{n+1}} x_{1}^{\beta_{1}} \cdot \ldots \cdot x_{n+1}^{\beta_{n+1}}
\end{aligned}
$$

be polynomials in $B\left[x_{1}, \ldots, x_{n+1}\right]$. Let

$$
h\left(x_{1}, \ldots, x_{n+1}\right)=f\left(x_{1}, \ldots, x_{n+1}\right) g\left(x_{1}, \ldots, x_{n+1}\right) .
$$

Thus $h\left(x_{1}, \ldots, x_{n+1}\right)$ has the form $\sum_{\gamma_{1}+\cdots+\gamma_{n+1}=0}^{m+p} s_{\gamma_{1}, \ldots, \gamma_{n+1}} x_{1}^{\gamma_{1}} \cdot \ldots \cdot x_{n+1}^{\gamma_{n+1}}$, where $s_{\gamma_{1}, \ldots, \gamma_{n+1}}$ are the indicated coefficients in $K[U, V]$.

Lemma 1.1. Let $z, t$ be non-zero elements in the $K\left[x_{1}, \ldots, x_{n}\right]$-submodule of $B\left[x_{1}, \ldots, x_{n+1}\right]$ generated by $U \cup V$. If $z t$ is in the $K\left[x_{1}, \ldots, x_{n}\right]$-submodule generated by $s_{\gamma_{1}, \ldots, \gamma_{n+1}}\left(0 \leq \gamma_{1}+\cdots+\gamma_{n+1} \leq m+p\right)$, then either $z \in K\left[U, x_{1}, \ldots, x_{n}\right]$ and $t \in K\left[V, x_{1}, \ldots, x_{n}\right]$, or $z \in K\left[V, x_{1}, \ldots, x_{n}\right]$ and $t \in K\left[U, x_{1}, \ldots, x_{n}\right]$.

Proof. This follows from the fact that the $s_{\gamma_{1}, \ldots, \gamma_{n+1}}$ are linear both in the indeterminates $U$ and in the indeterminates $V$.

Lemma 1.2. Let $z$ be in the $K\left[x_{1}, \ldots, x_{n}\right]$-submodule of $B\left[x_{1}, \ldots, x_{n+1}\right]$ generated by $U$ and let $t$ be in the $K\left[x_{1}, \ldots, x_{n}\right]$-submodule generated by $V$. If $z$ and $t$ are non-zero then the following conditions are equivalent:

(i) zt is in the $K\left[x_{1}, \ldots, x_{n}\right]$-submodule generated by $s_{\gamma_{1}, \ldots, \gamma_{n+1}}\left(0 \leq \gamma_{1}+\cdots+\right.$ $\left.\gamma_{n+1} \leq m+p\right)$.

(ii) Either $z t$ is in the $K\left[x_{1}, \ldots, x_{n}\right]$-submodule generated by $s_{\gamma_{1}, \ldots, \gamma_{n+1}}$, where $\gamma_{1}+\cdots+\gamma_{n+1}=m+p$, or there exist $c_{0}, c_{1}, \ldots, c_{n+1}, d_{0} \in K\left[x_{1}, \ldots, x_{n}\right]$ such that $z=c_{0} f\left(\frac{c_{1}}{c_{0}}, \ldots, \frac{c_{n+1}}{c_{0}}\right)$ and $t=d_{0} g\left(\frac{c_{1}}{c_{0}}, \ldots, \frac{c_{n+1}}{c_{0}}\right)$.

(iii) Either $z t$ is in the $K\left[x_{1}, \ldots, x_{n}\right]$-submodule generated by $s_{\gamma_{1}, \ldots, \gamma_{n+1}}$, where $\gamma_{1}+\cdots+\gamma_{n+1}=m+p$, or there exist $c_{0}, c_{1}, \ldots, c_{n+1}, d_{0} \in K\left[x_{1}, \ldots, x_{n}\right]$ such that $z t=c_{0} d_{0} h\left(\frac{c_{1}}{c_{0}}, \ldots, \frac{c_{n+1}}{c_{0}}\right)$.

Proof. (ii) $\Rightarrow$ (iii) and (iii) $\Rightarrow$ (i) are obvious.

(i) $\Rightarrow$ (ii). Let

$$
\begin{aligned}
& z=\sum_{\alpha_{1}+\cdots+\alpha_{n+1}=0}^{m} \lambda_{\alpha_{1}, \ldots, \alpha_{n+1}} u_{\alpha_{1}, \ldots, \alpha_{n+1}}, \\
& t=\sum_{\beta_{1}, \ldots, \beta_{n+1}=0}^{p} \mu_{\beta_{1}, \ldots, \beta_{n+1}} v_{\beta_{1}, \ldots, \beta_{n+1}},
\end{aligned}
$$


and

$$
z t=\sum_{\gamma_{1}+\cdots+\gamma_{n+1}=0}^{m+p} \nu_{\gamma_{1}, \ldots, \gamma_{n+1}} s_{\gamma_{1}, \ldots, \gamma_{n+1}},
$$

where $\lambda, \mu, \nu \in K\left[x_{1}, \ldots, x_{n}\right]$.

Let $\alpha_{1}^{0}, \ldots, \alpha_{n+1}^{0}$ be such that $\lambda_{\alpha_{1}^{0}, \ldots, \alpha_{n+1}^{0}} \neq 0$ and $\alpha_{1}^{0}+\cdots+\alpha_{n+1}^{0}$ is the least possible. Let $\beta_{1}^{0}, \ldots, \beta_{n+1}^{0}$ be such that $\mu_{\beta_{1}^{0}, \ldots, \beta_{n+1}^{0}} \neq 0$ and $\beta_{1}^{0}+\cdots+\beta_{n+1}^{0}$ is the least possible. If $\alpha_{1}^{0}+\cdots+\alpha_{n+1}^{0}+\beta_{1}^{0}+\cdots+\beta_{n+1}^{0}=m+p$ then $z t=$ $\sum_{\gamma_{1}+\cdots+\gamma_{n+1}=m+p} \nu_{\gamma_{1}, \ldots, \gamma_{n+1}} s_{\gamma_{1}, \ldots, \gamma_{n+1}}$. Suppose that $\alpha_{1}^{0}+\cdots+\alpha_{n+1}^{0}+\beta_{1}^{0}+\cdots+$ $\beta_{n+1}^{0}<m+p$. In this case we shall see that $\alpha_{1}^{0}=\cdots=\alpha_{n+1}^{0}=\beta_{1}^{0}=\cdots=\beta_{n+1}^{0}=$ 0 . Suppose that $\alpha_{i}^{0}>0$ for some $i$. If $\beta_{1}^{0}+\cdots+\beta_{n+1}^{0}<p$, then

$$
u_{\alpha_{1}^{0}, \ldots, \alpha_{i}^{0}-1, \ldots, \alpha_{n+1}^{0}} v_{\beta_{1}^{0}, \ldots, \beta_{i}^{0}+1, \ldots, \beta_{n+1}^{0}}
$$

appears in $s_{\alpha_{1}^{0}+\beta_{1}^{0}, \ldots, \alpha_{n+1}^{0}+\beta_{n+1}^{0}}$. Thus $\lambda_{\alpha_{1}^{0}, \ldots, \alpha_{i}^{0}-1, \ldots, \alpha_{n+1}^{0}} \neq 0$, in contradiction with the selection of $\alpha_{1}^{0}, \ldots, \alpha_{n+1}^{0}$. Hence $\beta_{1}^{0}+\cdots+\beta_{n+1}^{0}=p$. Now $\beta_{j}^{0}>0$ for some $j$ and $\alpha_{1}^{0}+\cdots+\alpha_{n+1}^{0}<m$. As above, we have a contradiction. Thus $\alpha_{i}^{0}=0$ for all $i$, and by symmetry $\beta_{j}^{0}=0$ for all $j$. Note that then $\lambda_{0, \ldots, 0} \neq 0$ and $\mu_{0, \ldots, 0} \neq 0$. Now

In this case, let $c_{0}=\lambda_{0, \ldots, 0}, c_{1}=\lambda_{1,0, \ldots, 0}, \ldots, c_{n+1}=\lambda_{0, \ldots, 0,1}, d_{0}=\mu_{0, \ldots, 0}$.

$$
\begin{aligned}
& \nu_{1,0, \ldots, 0}=c_{0} \mu_{1,0, \ldots, 0}=c_{1} d_{0}, \\
& \ldots \\
& \nu_{0, \ldots, 0,1}=c_{0} \mu_{0, \ldots, 0,1}=c_{n+1} d_{0} .
\end{aligned}
$$

If $\beta_{1}+\ldots+\beta_{n+1} \geq 2$, then there exists $i$ such that $\beta_{i} \geq 1$ and

$$
\nu_{\beta_{1}, \ldots, \beta_{n+1}}=\lambda_{0, \ldots, 0} \mu_{\beta_{1}, \ldots, \beta_{n+1}}=\lambda_{0, \ldots, 1, \ldots, 0} \mu_{\beta_{1}, \ldots, \beta_{i}-1, \ldots \beta_{n+1}}
$$

hence

$$
\mu_{\beta_{1}, \ldots, \beta_{n+1}}=\frac{c_{i}}{c_{0}} \mu_{\beta_{1}, \ldots, \beta_{i}-1, \ldots \beta_{n+1}} .
$$

Now, by induction on $\beta_{1}+\cdots+\beta_{n+1}$, we see that

$$
\mu_{\beta_{1}, \ldots, \beta_{n+1}}=\frac{c_{1}^{\beta_{1}} \cdot \ldots \cdot c_{n+1}^{\beta_{n+1}}}{c_{0}^{\beta_{1}+\cdots+\beta_{n+1}}} d_{0} .
$$

In a similar way it is easy to see that

$$
\lambda_{\alpha_{1}, \ldots, \alpha_{n+1}}=\frac{c_{1}^{\alpha_{1}} \cdot \ldots \cdot c_{n+1}^{\alpha_{n+1}}}{c_{0}^{\alpha_{1}+\cdots+\alpha_{n+1}}} c_{0} .
$$

Thus $z=c_{0} f\left(\frac{c_{1}}{c_{0}}, \ldots, \frac{c_{n+1}}{c_{0}}\right)$ and $t=d_{0} g\left(\frac{c_{1}}{c_{0}}, \ldots, \frac{c_{n+1}}{c_{0}}\right)$.

Let $u_{\alpha_{1}, \ldots, \alpha_{n+1}}^{(m)}, v_{\beta_{1}, \ldots, \beta_{n+1}}^{(m)}$ be indeterminates over $K$ for $m \geq 1,0 \leq \alpha_{1}+\cdots+$ $\alpha_{n+1} \leq d_{m}, 0 \leq \beta_{1}+\cdots+\beta_{m+1} \leq e_{m}$, where $d_{m} \geq 1, e_{m} \geq 1$. Let

$$
\begin{aligned}
& \mathcal{U}=\left\{u_{\alpha_{1}, \ldots, \alpha_{n+1}}^{(m)} \mid m \geq 1,0 \leq \alpha_{1}+\cdots+\alpha_{n+1} \leq d_{m}\right\}, \\
& \mathcal{V}=\left\{v_{\beta_{1}, \ldots, \beta_{n+1}}^{(m)} \mid m \geq 1,0 \leq \beta_{1}+\cdots+\beta_{n+1} \leq e_{m}\right\}
\end{aligned}
$$


Let $\mathcal{B}=K[\mathcal{U}, \mathcal{V}]$ and

$$
\begin{gathered}
f_{m}=f_{m}\left(x_{1}, \ldots, x_{n+1}\right)=\sum_{\alpha_{1}+\cdots+\alpha_{n+1}=0}^{d_{m}} u_{\alpha_{1}, \ldots, \alpha_{n+1}}^{(m)} x_{1}^{\alpha_{1}} \cdot \ldots \cdot x_{n+1}^{\alpha_{n+1}}, \\
g_{m}=g_{m}\left(x_{1}, \ldots, x_{n+1}\right)=\sum_{\beta_{1}+\cdots+\beta_{n+1}=0}^{e_{m}} v_{\beta_{1}, \ldots, \beta_{n+1}}^{(m)} x_{1}^{\beta_{1}} \cdot \ldots \cdot x_{n+1}^{\beta_{n+1}}, \\
h_{m, p}\left(x_{1}, \ldots, x_{n+1}\right)=f_{m} g_{p}=\sum_{\gamma_{1}+\cdots+\gamma_{n+1}=0}^{d_{m}+e_{p}} s_{\gamma_{1}, \ldots, \gamma_{n+1}}^{(m, p)} x_{1}^{\gamma_{1}} \cdot \ldots \cdot x_{n+1}^{\gamma_{n+1}} .
\end{gathered}
$$

Let $\mathcal{C}$ be the $K\left[x_{1}, \ldots, x_{n}\right]$-submodule of $\mathcal{B}\left[x_{1}, \ldots, x_{n+1}\right]$ generated by $s_{\gamma_{1}, \ldots, \gamma_{n+1}}^{(m, p)}$ for $0 \leq \gamma_{1}+\cdots+\gamma_{n+1} \leq d_{m}+e_{p}$ and for all $m, p \geq 1$.

Lemma 1.3. Let $p_{1}=\sum \lambda_{m} f_{m}+\sum \lambda_{m}^{\prime} g_{m}$ and $p_{2}=\sum \mu_{m} f_{m}+\sum \mu_{m}^{\prime} g_{m}$ be in $\mathcal{B}\left[x_{1}, \ldots, x_{n+1}\right] \backslash\{0\}$, where $\lambda, \lambda^{\prime}, \mu, \mu^{\prime} \in K\left[x_{1}, \ldots, x_{n}\right]$.

Let $c_{1}, \ldots, c_{n+1}, c_{1}^{\prime}, \ldots, c_{n+1}^{\prime} \in K\left(x_{1}, \ldots, x_{n}\right)$. Suppose that

$$
\begin{aligned}
& \sum \lambda_{m} f_{m}\left(c_{1}, \ldots, c_{n+1}\right)+\sum \lambda_{m}^{\prime} g_{m}\left(c_{1}, \ldots, c_{n+1}\right) \\
= & \sum \mu_{m} f_{m}\left(c_{1}^{\prime}, \ldots, c_{n+1}^{\prime}\right)+\sum \mu_{m}^{\prime} g_{m}\left(c_{1}^{\prime}, \ldots, c_{n+1}^{\prime}\right) .
\end{aligned}
$$

Then $p_{1}=p_{2}$ and $c_{i}=c_{i}^{\prime}$ for all $i$.

Proof. Since the coefficients of $f_{m}\left(x_{1}, \ldots, x_{n+1}\right)$ and $g_{m}\left(x_{1}, \ldots, x_{n+1}\right)$ are independent over $K\left[x_{1}, \ldots, x_{n+1}\right]$, we have

$$
\begin{aligned}
\lambda_{m} f_{m}\left(c_{1}, \ldots, c_{n+1}\right) & =\mu_{m} f_{m}\left(c_{1}^{\prime}, \ldots, c_{n+1}^{\prime}\right), \\
\lambda_{m}^{\prime} g_{m}\left(c_{1}, \ldots, c_{m}\right) & =\mu_{m}^{\prime} g_{m}\left(c_{1}^{\prime}, \ldots, c_{n+1}^{\prime}\right)
\end{aligned}
$$

for all $m \geq 1$.

Now $\lambda_{m} u_{0, \ldots, 0}^{(m)}=\mu_{m} u_{0, \ldots, 0}^{(m)}$ and $\lambda_{m}^{\prime} v_{0, \ldots, 0}^{(m)}=\mu_{m}^{\prime} v_{0, \ldots, 0}^{(m)}$. Thus $\lambda_{m}=\mu_{m}$ and $\lambda_{m}^{\prime}=\mu_{m}^{\prime}$. Hence $p_{1}=p_{2}$.

Without lost of generality we may assume that $\lambda_{m} \neq 0$. Then

$$
\lambda_{m} c_{i} u_{0, \ldots, 1, \ldots, 0}^{(m)}=\mu_{m} c_{i}^{\prime} u_{0, \ldots, 1, \ldots, 0}^{(m)} .
$$

Thus $c_{i}=c_{i}^{\prime}$ for all $i$.

The next lemma is an easy generalization of Lemma 1.1.

Lemma 1.4. Let $z, t$ be non-zero elements in the $K\left[x_{1}, \ldots, x_{n}\right]$-submodule of $\mathcal{B}\left[x_{1}, \ldots, x_{n+1}\right]$ generated by $\mathcal{U} \cup \mathcal{V}$. If $z t \in \mathcal{C}$, then either $z \in K\left[\mathcal{U}, x_{1}, \ldots, x_{n}\right]$ and $t \in K\left[\mathcal{V}, x_{1}, \ldots, x_{n}\right]$, or $z \in K\left[\mathcal{V}, x_{1}, \ldots, x_{n}\right]$ and $t \in K\left[\mathcal{U}, x_{1}, \ldots, x_{n}\right]$.

Lemma 1.5. Let $z$ be in the $K\left[x_{1}, \ldots, x_{n}\right]$-submodule of $\mathcal{B}\left[x_{1}, \ldots, x_{n+1}\right]$ generated by $\mathcal{U}$ and let $t$ be in the $K\left[x_{1}, \ldots, x_{n}\right]$-submodule generated by $\mathcal{V}$. Then the following conditions are equivalent:

(i) $z t \in \mathcal{C}$.

(ii) Either $z t$ is in the $K\left[x_{1}, \ldots, x_{n}\right]$-submodule generated by $s_{\gamma_{1}, \ldots, \gamma_{n+1}}^{(m, p)}$, where $\gamma_{1}, \ldots, \gamma_{n+1}=d_{m}+e_{p}$, or there exist $\lambda_{m}, \mu_{m} \in K\left[x_{1}, \ldots, x_{n}\right]$ and $c_{1}, \ldots$, $c_{n+1} \in K\left(x_{1}, \ldots, x_{n}\right)$ such that

$$
z=\sum \lambda_{m} f_{m}\left(c_{1}, \ldots, c_{n+1}\right) \text { and } t=\sum \mu_{m} g_{m}\left(c_{1}, \ldots, c_{n+1}\right) .
$$


In fact, in (ii) $\lambda_{m} f_{m}\left(c_{1}, \ldots, c_{n+1}\right)$ and $\mu_{m} g_{m}\left(c_{1}, \ldots, c_{n+1}\right) \in \mathcal{B}\left[x_{1}, \ldots, x_{n}\right]$.

Proof. (ii) $\Rightarrow$ (i) is obvious.

(i) $\Rightarrow$ (ii) The element $z$ has a unique representation as a sum $z=\sum_{m} z^{(m)}$, where $z^{(m)}$ is in the $K\left[x_{1}, \ldots, x_{n}\right]$-submodule generated by $u_{\alpha_{1}, \ldots, \alpha_{n+1}}^{(m)}\left(0 \leq \alpha_{1}+\cdots+\right.$ $\left.\alpha_{n+1} \leq d_{m}\right)$. Similarly $t=\sum_{m} t^{(m)}$, where $t^{(m)}$ is in the $K\left[x_{1}, \ldots, x_{n}\right]$-submodule generated by $v_{\beta_{1}, \ldots, \beta_{n+1}}^{(m)}\left(0 \leq \beta_{1}+\cdots+\beta_{n+1} \leq e_{m}\right)$.

Now it is easy to see that since $z t \in \mathcal{C}, z^{(m)} t^{(p)}$ is in the $K\left[x_{1}, \ldots, x_{n}\right]$-submodule generated by $s_{\gamma_{1}, \ldots, \gamma_{n+1}}^{(m, p)}\left(0 \leq \gamma_{1}+\cdots+\gamma_{n+1} \leq d_{m}+e_{p}\right)$. Suppose that $z^{(m)}$ and $t^{(p)}$ are non-zero. By Lemma 1.2, either $z^{(m)} t^{(p)}$ is in the $K\left[x_{1}, \ldots, x_{n}\right]$-submodule generated by $s_{\gamma_{1}, \ldots, \gamma_{n+1}}^{(m, p)}$, where $\gamma_{1}+\cdots+\gamma_{n+1}=d_{m}+e_{p}$, or there exist $\lambda_{m, p}, \mu_{m, p}$, $c_{1}^{(m, p)}, \ldots, c_{n+1}^{(m, p)} \in K\left[x_{1}, \ldots, x_{n}\right]$ such that

$$
\begin{aligned}
z^{(m)} & =\lambda_{m, p} f_{m}\left(\frac{c_{1}^{(m, p)}}{\lambda_{m, p}}, \ldots, \frac{c_{n+1}^{(m, p)}}{\lambda_{m, p}}\right), \\
t^{(p)} & =\mu_{m, p} g_{p}\left(\frac{c_{1}^{(m, p)}}{\lambda_{m, p}}, \ldots, \frac{c_{n+1}^{(m, p)}}{\lambda_{m, p}}\right) .
\end{aligned}
$$

Furthermore, in the latter case, if $z^{\left(m^{\prime}\right)} \neq 0$ then there exist $\lambda_{m^{\prime}, p}, \mu_{m^{\prime}, p}, c_{1}^{\left(m^{\prime}, p\right)}, \ldots$, $c_{n+1}^{\left(m^{\prime}, p\right)} \in K\left[x_{1}, \ldots, x_{n}\right]$ such that

$$
\begin{aligned}
z^{\left(m^{\prime}\right)} & =\lambda_{m^{\prime}, p} f_{m^{\prime}}\left(\frac{c_{1}^{\left(m^{\prime}, p\right)}}{\lambda_{m^{\prime}, p}}, \ldots, \frac{c_{n+1}^{\left(m^{\prime}, p\right)}}{\lambda_{m^{\prime}, p}}\right), \\
t^{(p)} & =\mu_{m^{\prime}, p} g_{p}\left(\frac{c_{1}^{\left(m^{\prime}, p\right)}}{\lambda_{m^{\prime}, p}}, \ldots, \frac{c_{n+1}^{\left(m^{\prime}, p\right)}}{\lambda_{m^{\prime}, p}}\right) .
\end{aligned}
$$

By Lemma 1.3, $\mu_{m, p}=\mu_{m^{\prime}, p}$ and $\frac{c_{i}^{(m, p)}}{\lambda_{m, p}}=\frac{c_{i}^{\left(m^{\prime}, p\right)}}{\lambda_{m^{\prime}, p}}$. Thus $\mu_{m, p}$ depends just on $p$ and not on $m$, so we set $\mu_{m, p}=\mu_{p}$ for all $m$ such that $t^{(p)} \neq 0$. Similarly, $\lambda_{m, p}$ depends just on $m$ and not on $p$, so we set $\lambda_{m, p}=\lambda_{m}$. We have seen that $\frac{c_{i}^{(m, p)}}{\lambda_{m, p}}$ does not depend on $m$ and similarly for $p$, so we set $\frac{c_{i}^{(m, p)}}{\lambda_{m, p}}=c_{i}$. Hence

$$
z=\sum_{m} \lambda_{m} f_{m}\left(c_{1}, \ldots, c_{n+1}\right) \text { and } t=\sum_{p} \mu_{p} g_{p}\left(c_{1}, \ldots, c_{n+1}\right) .
$$

\section{The RESUlT}

In this section we assume that $K$ is a field with $|K| \leq \aleph_{0}$. Thus

$$
\left|K\left(x_{1}, \ldots, x_{n}\right)^{n+1}\right| \leq \aleph_{0} .
$$

Given $c \in K\left(x_{1}, \ldots, x_{n}\right)$, we denote by $p_{c}$ and $q_{c}$ two coprime elements of $K\left[x_{1}, \ldots, x_{n}\right]$ such that $c=p_{c} / q_{c}$. Let $\left(c_{1,1}, \ldots, c_{1, n+1}\right),\left(c_{2,1}, \ldots, c_{2, n+1}\right), \ldots$, $\left(c_{m, 1}, \ldots, c_{m, n+1}\right), \ldots$ be the sequence of all elements in $K\left(x_{1}, \ldots, x_{n}\right)^{n+1}$. As in the above section we define $\mathcal{U}, \mathcal{V}, \mathcal{B}, f_{m}, g_{m}$ and $\mathcal{C}$. Let $c_{m, 0}$ be the least common 
multiple of $q_{c_{m, 1}}, \ldots, q_{c_{m, n+1}}$, so $c_{m, 0} c_{m, j} \in K\left[x_{1}, \ldots, x_{n}\right]$ for $1 \leq j \leq n+1$. For $m \geq 1$ and $i \geq 1$, let $a_{m i}$ be a non-zero polynomial of least degree in $K\left[x_{1}, \ldots, x_{n}\right]$ such that $a_{m i} f_{m}\left(c_{i, 1}, \ldots, c_{i, n+1}\right) g_{m}\left(c_{i, 1}, \ldots, c_{i, n+1}\right) \in \mathcal{B}\left[x_{1}, \ldots, x_{n}\right]$. Note that the degree of $a_{m i}$ is $\leq \operatorname{deg}\left(c_{i, 0}\right) \cdot\left(d_{m}+e_{m}\right)$.

Let $I$ be the ideal of $\mathcal{B}$ generated by $s_{\gamma_{1}, \ldots, \gamma_{n+1}}^{(m, p)}$ for all $m \neq p$, the coefficients of the polynomials

$$
a_{m_{i}} f_{m}\left(c_{i, 1}, \ldots, c_{i, n+1}\right) g_{m}\left(c_{i, 1}, \ldots, c_{i, n+1}\right)
$$

for all $1 \leq i<m$, the elements $s_{\gamma_{1}, \ldots, \gamma_{n+1}}^{(m, m)}$ for $\gamma_{1}+\cdots+\gamma_{n+1}=d_{m}+e_{m}$ and all monomials in the indeterminates $\mathcal{U} \cup \mathcal{V}$ of degree 3 . Let $\mathcal{R}$ be the $\operatorname{ring} \mathcal{B} / I$. Thus $\mathcal{R}$ has a natural grading inherited from $\mathcal{B}, \mathcal{R}=\mathcal{R}_{0} \oplus \mathcal{R}_{1} \oplus \mathcal{R}_{2}$, and $\mathcal{R}_{0}=K$. We denote by $\bar{r}$ the canonical image of $r \in \mathcal{B}\left[x_{1}, \ldots, x_{n+1}\right]$ in $\mathcal{R}\left[x_{1}, \ldots, x_{n+1}\right]$.

Lemma 2.1. The ring $\mathcal{R}\left[x_{1}, \ldots, x_{n}\right]$ satisfies acc $\perp$.

Proof. To prove that $\mathcal{R}\left[x_{1}, \ldots, x_{n}\right]$ satisfies acc $\perp$ it is enough to show that for any pair of sequences $\left\{z_{m}\right\}_{m \geq 1}$ and $\left\{t_{m}\right\}_{m \geq 1}$ of nonzero elements in $\mathcal{B}_{1}\left[x_{1}, \ldots, x_{n}\right]$ such that $\bar{z}_{m} \bar{t}_{p}=0$ for $1 \leq p<m$, we have $\bar{z}_{m} \bar{t}_{m}=0$ for almost all $m$.

For $m>p$ the elements $z_{m} t_{p}$ are in $I_{2}\left[x_{1}, \ldots, x_{n}\right] \subseteq \mathcal{C}$. By Lemma 1.4 we may assume that $z_{m} \in K\left[\mathcal{U}, x_{1}, \ldots, x_{n}\right]$ and $t_{p} \in K\left[\mathcal{V}, x_{1}, \ldots, x_{n}\right]$ for all $p, m \geq 1$.

By Lemma 1.5 there exist $\lambda_{k, m, p}, \mu_{k, m, p} \in K\left[x_{1}, \ldots, x_{n}\right] \backslash\{0\}$, and $c_{1, m, p}, \ldots$, $c_{n+1, m, p} \in K\left(x_{1}, \ldots, x_{n}\right)$ such that

$$
\begin{aligned}
z_{m} & =\sum_{k} \lambda_{k, m, p} f_{k}\left(c_{1, m, p}, \ldots, c_{n+1, m, p}\right) \text { and } \\
t_{p} & =\sum_{k} \mu_{k, m, p} g_{k}\left(c_{1, m, p}, \ldots, c_{n+1, m, p}\right)
\end{aligned}
$$

with $\lambda_{k, m, p} f_{k}\left(c_{1, m, p}, \ldots, c_{n+1, m, p}\right), \mu_{k, m, p} g_{k}\left(c_{1, m, p}, \ldots, c_{n+1, m, p}\right) \in \mathcal{B}\left[x_{1}, \ldots, x_{n}\right]$.

By Lemma 1.3, $\lambda_{k, m, p}$ does not depend on $p, \mu_{k, m, p}$ does not depend on $m$ and $c_{i, m, p}$ depends on neither $m$ nor $p$. So we set $\lambda_{k, m, p}=\lambda_{k, m}, \mu_{k, m, p}=\mu_{k, p}$ and $c_{i, m, p}=c_{i}$. Thus

$$
\begin{aligned}
z_{m} & =\sum_{k} \lambda_{k, m} f_{k}\left(c_{1}, \ldots, c_{n+1}\right) \text { and } \\
t_{p} & =\sum_{k} \mu_{k, p} g_{k}\left(c_{1}, \ldots, c_{n+1}\right)
\end{aligned}
$$

Choose $j$ such that $\left(c_{1}, \ldots, c_{n+1}\right)=\left(c_{j, 1}, \ldots, c_{j, n+1}\right)$. Let

$$
\begin{aligned}
z_{m}^{\prime} & =\sum_{k=1}^{j} \lambda_{k, m} f_{k}\left(c_{1}, \ldots, c_{n+1}\right) \text { and } \\
t_{p}^{\prime} & =\sum_{k=1}^{j} \mu_{k, p} g_{k}\left(c_{1}, \ldots, c_{n+1}\right) .
\end{aligned}
$$

Let $k>j$. Since $a_{k j}$ is the polynomial of minimal degree such that

$$
a_{k j} f_{k}\left(c_{j, 1}, \ldots, c_{j, n+1}\right) g_{k}\left(c_{j, 1}, \ldots, c_{j, n+1}\right) \in \mathcal{B}\left[x_{1}, \ldots, x_{n}\right]
$$

and $f_{k} g_{k^{\prime}} \in I\left[x_{1}, \ldots, x_{n+1}\right]$ for all $k \neq k^{\prime}$, we have that $\bar{z}_{m} \bar{t}_{p}$ is the canonical image of $z_{m}^{\prime} t_{p}^{\prime}$. 
Now there exists $p_{0}$ such that for any $p \geq p_{0}, t_{p}^{\prime}$ is a $K\left(x_{1}, \ldots, x_{n}\right)$-linear combination of $t_{1}^{\prime}, \ldots, t_{p_{0}-1}^{\prime}$. Thus for each $p \geq p_{0}$ there exist $b_{1, p}, \ldots, b_{p_{0}-1, p} \in$ $K\left(x_{1}, \ldots, x_{n}\right)$ such that

$$
t_{p}^{\prime}=\sum_{l=1}^{p_{0}-1} b_{l, p} t_{l}^{\prime}
$$

Hence for any $p \geq p_{0}$

$$
z_{p}^{\prime} t_{p}^{\prime}=\sum_{l=1}^{p_{0}-1} b_{l, p} t_{l}^{\prime} z_{p}^{\prime} \in I\left(x_{1}, \ldots, x_{n}\right) \cap \mathcal{B}\left[x_{1}, \ldots, x_{n}\right]=I\left[x_{1}, \ldots, x_{n}\right] .
$$

Therefore $\bar{z}_{p} \bar{t}_{p}=0$ for any $p \geq p_{0}$.

Assume $m>1$ and let $l_{m}$ be the maximum of the degrees of $c_{j 0}$, for $1 \leq j<m$. Let $l_{m}^{\prime}$ be the maximum of the degrees of $c_{j 0} c_{j i}$, for $1 \leq j<m$ and $1 \leq i \leq n+1$. Hence, the degree of $a_{m j} f_{m}\left(c_{j, 1}, \ldots, c_{j, n+1}\right) g_{m}\left(c_{j, 1}, \ldots, c_{j, n+1}\right)$ is $\leq l_{m}\left(d_{m}+e_{m}\right)+$ $l_{m}^{\prime}\left(d_{m}+e_{m}\right)=\left(l_{m}+l_{m}^{\prime}\right)\left(d_{m}+e_{m}\right)$. Thus the number of non-zero coefficients of this polynomial, as an element of $\mathcal{B}\left[x_{1}, \ldots, x_{n}\right]$, is $\leq\left(\begin{array}{c}n+\left(l_{m}+l_{m}^{\prime}\right)\left(d_{m}+e_{m}\right) \\ \left(l_{m}+l_{m}^{\prime}\right)\left(d_{m}+e_{m}\right)\end{array}\right)$.

Note that the number of coefficients of $f_{m} g_{m} \in \mathcal{B}\left[x_{1}, \ldots, x_{n+1}\right]$ is

$$
\left(\begin{array}{c}
n+1+d_{m}+e_{m} \\
d_{m}+e_{m}
\end{array}\right)
$$

Observe that $\left(\begin{array}{c}n+1+d_{m}+e_{m} \\ d_{m}+e_{m}\end{array}\right)$ is a polynomial of degree $(n+1)$ ! in $d_{m}+e_{m}$ while $\left(\begin{array}{c}n+\left(l_{m}+l_{m}^{\prime}\right)\left(d_{m}+e_{m}\right) \\ \left(l_{m}+l_{m}^{\prime}\right)\left(d_{m}+e_{m}\right)\end{array}\right)$ is a polynomial of degree $n$ ! in $d_{m}+e_{m}$. Now it is clear that for each $m>1$ there exists a positive integer $N_{m}$ such that if $d_{m}+e_{m} \geq N_{m}$ then

$$
\left(\begin{array}{c}
n+1+d_{m}+e_{m} \\
d_{m}+e_{m}
\end{array}\right)>m\left(\begin{array}{c}
n+\left(l_{m}+l_{m}^{\prime}\right)\left(d_{m}+e_{m}\right) \\
\left(l_{m}+l_{m}^{\prime}\right)\left(d_{m}+e_{m}\right)
\end{array}\right)
$$

Proposition 2.2. Suppose that $d_{m}+e_{m} \geq N_{m}$ for all $m>1$. Then the ring $\mathcal{R}\left[x_{1}, \ldots, x_{n+1}\right]$ does not satisfy acc $\perp$.

Proof. Clearly, $\bar{f}_{m} \bar{g}_{p}=0$ for $m \neq p$. On the other hand, for any $m$, the $K$-subspace $W_{m}$ generated by the coefficients of $f_{m} g_{m}$ has dimension $\left(\begin{array}{c}n+1+d_{m}+e_{m} \\ d_{m}+e_{m}\end{array}\right)$ and $W_{m} \cap I$ is generated by the coefficients of $a_{m i} f_{m}\left(c_{i, 1}, \ldots, c_{i, n+1}\right) g\left(c_{i, 1}, \ldots, c_{i, n+1}\right)$ for all $1 \leq i<m$. So

$$
\operatorname{dim}_{K}\left(W_{m} \cap I\right) \leq m\left(\begin{array}{c}
n+\left(l_{m}+l_{m}^{\prime}\right)\left(d_{m}+e_{m}\right) \\
\left(l_{m}+l_{m}^{\prime}\right)\left(d_{m}+e_{m}\right)
\end{array}\right) .
$$

Since $d_{m}+e_{m} \geq N_{m}$ for all $m>1$, we have

$$
\operatorname{dim}_{K}\left(W_{m} \cap I\right)<\operatorname{dim}_{K} W_{m}
$$

for all $m>1$. Thus $f_{m} g_{m} \notin I\left[x_{1}, \ldots, x_{n+1}\right]$ and $\bar{f}_{m} \bar{g}_{m} \neq 0$ for all $m>1$. Hence $\mathcal{R}\left[x_{1}, \ldots, x_{n+1}\right]$ does not satisfy acc $\perp$. 


\section{REFERENCES}

1. V. Camillo and R. Guralnick, Polynomial rings over Goldie rings are often Goldie, Proc. A.M.S. 98 (1986), 567-568. MR 87k:16018

2. C. Faith, Polynomial rings over Goldie-Kerr commutative rings, Proc. A.M.S. 120 (1994), 989-993. MR 94k:13024

3. J. W. Kerr, The polynomial ring over a Goldie ring need not be a Goldie ring, J. Alg. 134 (1990), 344-352. MR 91h:16042

4. M. Roitman, On polynomial extensions of Mori domains over countable fields, J. of Pure and Appl. Algebra 64 (1990), 315-328. MR 91i:13021

Departament de Matemàtiques, Universitat Autònoma de Barcelona, 08193 BellateRRA (BARCELONA), SPAin

E-mail address: cedo@mat.uab.es

E-mail address: dolors@mat.uab.es 